\title{
Constructing Phantom Cosmologies from Standard Scalar Field Universes
}

\author{
Luis P. Chimento ${ }^{1}$ and Ruth Lazkoz ${ }^{2}$ \\ 1 Dpto. de Física, Facultad de Ciencias Exactas y Naturales, \\ Universidad de Buenos Aires, Ciudad Universitaria \\ Pabellón I, 1428 Buenos Aires, Argentina \\ chimento@df.uba.ar \\ ${ }^{2}$ Fisika Teorikoa eta Zientziaren Historia Saila, Zientzia eta Teknologia Fakultatea, \\ Euskal Herriko Unibertsitatea, \\ 644 Posta Kutxatila, 48080 Bilbao, Spain \\ wtplasar@lg.ehu.es
}

\begin{abstract}
We illustrate how form-invariance transformations can be used for constructing phantom cosmologies from standard scalar field universes. First, we discuss how to relate two flat FriedmannRobertson-Walker cosmologies with different barotropic indexes $\gamma$ and $\bar{\gamma}$. Then, we consider the particular case $\bar{\gamma}=-\gamma$, and we show that if the matter content is interpreted in terms of selfinteracting scalar fields, then the corresponding transformation provides the link between a standard and a phantom cosmology. After that, we illustrate the method by considering models with exponential potentials. Finally, we also show that the mentioned duality persists even if the typical braneworld modifications to the Friedmann equation are considered.
\end{abstract}

Current observations can accommodate matter which violates the weak energy condition $\rho>0, \rho+p \geq 0$ [1]. One possible candidate for that sort of matter is a fluid with negative enough pressure. If the fluid has a barotropic equation of state of the form $p=(\gamma-1) \rho$, then $\gamma<1$. Alternatively, one can invoke matter of scalar nature so that the square of the time derivative of the scalar nature enters the energymomentum with an opposite sign compared to the standard situation. The cosmological models filled with that kind of matter are referred to as phantom or ghost cosmologies [2]. Even though the theoretical understanding of the subject is limited, we can rely on the motivation for phantom matter which is provided by string theory [3]. Moreover, we can rest assured that it does not go against the observations when considering such exotic matter. Those are the main reasons why phantom models have begun to attract the attention of an increasing number of theoreticians, including those concerned, with exact solutions [4, 5, 6] or cosmological dynamics [7]. There is also an interesting debate on what would be the fate of a universe with large negative pressure [8].

One of the ways to obtain new exact solutions of the Einstein field equations is to exploit their form-invariance symmetries. In cosmology, that method has been used [9, 10] for generating inflationary cosmologies from nonin- flationary ones. The Einstein equations for a flat Friedmann-Robertson-Walker (FRW) cosmological model with scale factor $a$, and filled with a perfect fluid with energy density $\rho$ and pressure $p$, are

$$
\begin{aligned}
& 3 H^{2}=\rho, \\
& \dot{\rho}+3 H(\rho+p)=0,
\end{aligned}
$$

where a dot denotes differentiation with respect to the cosmic time, and $H=\dot{a} / a$. The units used in the paper are such that $c=8 \pi G=1$.

A form-invariance transformation is a prescription to relate the quantities $a, H, \rho$ and $p$ of our original model, with the quantities $\bar{a}$, $\bar{H}, \bar{\rho}$ and $\bar{p}$ of what we will call the transformed model, so that it satisfies

$$
\begin{aligned}
& 3 \bar{H}^{2}=\bar{\rho} \\
& \dot{\bar{\rho}}+3 \bar{H}(\bar{\rho}+\bar{p})=0 .
\end{aligned}
$$

In [9] it was shown that one such transformation is given by

$$
\begin{aligned}
& \bar{\rho}=\bar{\rho}(\rho), \\
& \bar{H}=\left(\frac{\bar{\rho}}{\rho}\right)^{1 / 2} H \\
& \bar{p}=-\bar{\rho}+\left(\frac{\rho}{\bar{\rho}}\right)^{1 / 2}(\rho+p) \frac{d \bar{\rho}}{d \rho}
\end{aligned}
$$

where $\bar{\rho}=\bar{\rho}(\rho)$ is an invertible arbitrary function. 
Let us consider now perfect fluids with a barotropic equation of state $p=(\gamma-1) \rho$, where $\gamma$ is the so-called barotropic index. The barotropic indexes of the original and transformed fluid are related by

$$
\bar{\gamma}=\left(\frac{\rho}{\bar{\rho}}\right)^{3 / 2} \frac{d \bar{\rho}}{d \rho} \gamma
$$

A scalar field $\phi$ self-interacting through a potential $V(\phi)$ can also be interpreted in the language of perfect fluids. It is well know that one can make the following identifications:

$$
\begin{aligned}
& \rho=\frac{1}{2} \dot{\phi}^{2}+V(\phi), \\
& p=\frac{1}{2} \dot{\phi}^{2}-V(\phi) .
\end{aligned}
$$

Under the transformation

$$
\bar{\rho}=n^{2} \rho,
$$

$n$ being a constant, the scalar field and potential transform, respectively, as

$$
\begin{aligned}
& \dot{\bar{\phi}}^{2}=n \dot{\phi}^{2} \\
& \bar{V}=n^{2}\left(\frac{1}{2} \dot{\phi}^{2}+V\right)-\frac{n}{2} \dot{\phi}^{2},
\end{aligned}
$$

Let us now investigate the simple transformations which preserve the energy density of the fluid; that is, $\bar{\rho}=\rho$. From Eqs. (6) - (8) , we get

$$
\begin{aligned}
& \bar{H}= \pm H, \\
& \bar{p}=-\rho \pm(\rho+p) \\
& \bar{\gamma}= \pm \gamma .
\end{aligned}
$$

Clearly, the $(+)$ branch is the identical transformation. However, as will be shown immediately, the $(-)$ branch leads to a phantom symmetry of the FRW equations (11) and (2), because it corresponds to

$$
\bar{H}=-H, \quad \bar{\rho}+\bar{p}=-(\rho+p), \quad \bar{\gamma}=-\gamma .
$$

From these relations we see that the two quantities $\rho$ and $H \gamma$ are invariant under the phantom symmetry transformation.

On the other hand, the kinetic energy and the potential of the field transform as

$$
\begin{aligned}
& \dot{\bar{\phi}}^{2}=-\dot{\phi}^{2} \\
& \bar{V}(\bar{\phi})=\dot{\phi}^{2}+V(\phi),
\end{aligned}
$$

so in fact, up to a constant, we see that $\bar{\phi}=i \phi$. That is, the transformed scalar field is related to the original one by a Wick rotation, and we finally have

$$
\bar{\rho}=-\frac{1}{2} \dot{\phi}^{2}+\bar{V}(\bar{\phi})
$$

The sign in the kinetic part of the energy density indicates that what we actually have now is a phantom cosmology, with a phantom field $\phi$, together with a phantom potential $\bar{V}(\bar{\phi})$, which is a real function of $\phi$. Note that the selfinteraction potential in the phantom and standard cases are different, and are linked by the transformation rule (19).

Finally, in order to establish completely the link between flat FRW scalar field cosmologies with a barotropic equation of state and their phantom counterparts, we have to get $\bar{a}$ as a function of $a$. This follows directly from the relationship $\bar{H}=-H$, which gives in turn $\bar{a} \propto a^{-1}$. As we have seen, this duality, which was noticed independently in [4], is, in fact, a particular case of a more general form-invariance symmetry. Noticeably, this duality in the scale factor, which here allows one to establish a link between phantom and standard scalar field cosmologies, is identical to the one appearing in the pre-big bang models.

An straightforward extension would be the application of the method to the case of universes filled with several fluid components, or with multiple scalar fields, for instance in the fashion of [12], where an $O(\mathrm{~N})$ field configuration was considered. The details of how this generalization could be done can also be found in [9]. Nevertheless, for the sake of simplicity, here we will restrict the discussion to single field configurations.

For illustration purposes, let us take an exponential potential

$$
V=V_{0} \mathrm{e}^{-A \phi}
$$

where $A$ is a constant. As is well known, powerlaw solutions will exist if the scalar field has a logarithmic dependence on time. Such solutions to the Einstein-Klein-Gordon equation set will correspond to

$$
a=t^{2 / A^{2}}, \quad \phi=\frac{2}{A} \ln t, \quad V_{0}=\frac{2\left(6-A^{2}\right)}{A^{4}} .
$$

If we apply now the phantom symmetry given by Eq. (19), the transformed scalar field and 
potential is

$$
\bar{\phi}=i \frac{2}{A} \ln t, \quad \bar{V}=\bar{V}_{0} \mathrm{e}^{i A \bar{\phi}},
$$

with

$$
\bar{V}_{0}=\frac{2\left(6+A^{2}\right)}{A^{4}},
$$

so that the transformed scale factor reads

$$
\bar{a}=t^{-2 / A^{2}} .
$$

Therefore, it is clear that solutions (22) y (25) satisfy the symmetry condition $a \bar{a}=1$.

A question that immediately comes to mind is whether the form-invariance symmetry can be extended. To this end, the next line of attack is the extension to brane scenarios (see 13] for an study on brane cosmologies induced by phantom fields). The Friedmann equation for a perfect fluid obeying the standard conservation equation (2) on a flat FRW brane becomes

$$
3 H^{2}=\rho\left(1+\frac{\rho}{2 \lambda}\right)
$$

where bulk effects have been switched off, $\lambda$ being the brane tension.

The conservation equation (2) and the modified Friedmann equation (26) are form invariant under the symmetry transformation given by

$$
\bar{H}=\sqrt{\frac{\bar{\rho}(1+\bar{\rho} /(2 \lambda))}{\rho(1+\rho /(2 \lambda))}} H
$$

$$
\bar{p}=-\bar{\rho}+\sqrt{\frac{\bar{\rho}(1+\bar{\rho} /(2 \lambda))}{\rho(1+\rho /(2 \lambda))}}(\rho+p) \frac{d \bar{\rho}}{d \rho}
$$

It follows that, if we apply a symmetry transformation such that $\bar{\rho}=\rho$, we will obtain exactly the two same results as in the relativistic case, that is, $\bar{H}= \pm H$ and $\bar{a} \propto a^{ \pm 1}$, although the $(-)$ branch would correspond now to a brane phantom cosmology.

Summarizing, we have shown that there exists a duality between phantom and standard (or nonexotic) flat FRW cosmologies, both in the relativistic and braneworld frameworks. As a matter of fact, this duality is a particular case of the form-invariance symmetry of the corresponding gravitational field equations. It may be worth looking further into this problem, to see whether explicit relations could be obtained in less straightforward cases.

L.P.C. is supported by the University of Buenos Aires under project X223, and by the Consejo Nacional de Investigaciones Científicas y Técnicas. R.L. is supported by the Basque Government through fellowship BFI01.412, the Spanish Ministry of Science and Technology jointly with FEDER funds through research grant BFM2001-0988, and the University of the Basque Country through research grant UPV00172.310-14456/2002.
[1] R.R. Caldwell, Phys. Lett. B 545, 23 (2002); S. Hannestad and E. Mörstell, Phys. Rev. D 66, 063508 (2002); P.H. Frampton and T. Takahashi, Phys. Lett. B 557135 (2003); S. M. Carroll, M. Hoffman and M. Trodden, Phys. Rev. D 68, 023509 (2003); A. Melchiorri, L. Mersini, C. J. Odman, and M. Trodden, Phys. Rev. D 68043509 (2003).

[2] R.R. Caldwell, Phys. Lett. B545, 23, (2002); G.W. Gibbons, Phantom matter and the cosmological constant, hep-th/0302199

[3] L. Mersini, M. Bastero-Gil and P. Kanti, Phys. Rev. D 64043508 (2001); M. Bastero-Gil, P.H. Frampton, and L. Mersini, Phys. Rev. D 65106002 (2002); P.H. Frampton, Phys.Lett. B 555, 139 (2003).

[4] M.P. Dąbrowski, T. Stachowiak, and M. Szydłowski, Phantom cosmologies, hep-th/0307128
[5] S. Nojiri, S.D. Odintsov, Phys. Lett. B 562, 147 (2003).

[6] A. Feinstein, S. Jhingan, Ghosts in a mirror, hep-th/0304069

[7] P. Singh, M. Sami, and N. Dadhich, Phys. Rev. D 68, 023522 (2003).

[8] R. R. Caldwell, M. Kamionkowski, N.N. Weinberg, Phys. Rev. Lett. 91, 071301 (2003).; A. Yurov, Phantom scalar fields result in inflation rather than Big Rip, astro-ph/0305019 P.F. González-Díaz, Phys. Rev. D 68, 021303 (2003).

[9] L.P. Chimento, Phys. Rev. D 65, 063517 (2002).

[10] J.M. Aguirregabiria, L.P. Chimento, A. Jakubi, and Ruth Lazkoz, Phys. Rev. D 67, 083518 (2003).

[11] G. Veneziano, Phys. Lett. B 265, 287 (1991); 
K.A. Meissner and G. Veneziano, Phys. Lett. B 267, 33 (1991); Mod. Phys. Lett. A 6, 3397 (1991); J.E. Lidsey, D.W. Wands, and E.J. Copeland, Phys. Rep. 337, 343 (2000).

[12] Xin-zhou Li, Jian-gang Hao, O(N) phantom, a way to implement $\mathrm{w}<-1$, hep-th/0303093

[13] S. Nojiri, S.D. Odintsov, Phys. Lett. B 565, 1 (2003). 